\title{
Simulation of Unsteady Water Film Flow on Pelton Bucket
}

\author{
Shen Na \\ School of Electrical Engineering, Guangzhou College of South China University of Technology, Guangzhou, China \\ Email: nashen5@163.com
}

Received January, 2013

\begin{abstract}
In order to simulate the complicated unsteady flow in Pelton bucket, it is necessary to apply the animated cartoon approach. In this paper, a free jet and the inner surface of a bucket is described by boundary fitted grid (BFG) with non-orthogonal curvilinear coordinates. The water flow is discretized in space and time for CFD (computational fluid dynamics). The moving grids of water film are successfully projected onto the bucket's inner surface by a projection algorithm. The visualization result of the jet landing on bucket's surface and the unsteady flow in the rotating buckets in $3 \mathrm{D}$ verifies the effectiveness of the proposed method.
\end{abstract}

Keywords: Water Film Flow; Simulation; Projection; Pelton Bucket

\section{Introduction}

Different from the reaction turbines such as Francis and Kaplan turbines, the flow in Pelton turbine is essentially unsteady in space and time. This is the reason why the application of CFD (computational fluid dynamics) to the Pelton turbines is much behind other turbines. With the continuous improvement of computer technology and physical model, the numerical simulation of inner complicated flow in Pelton turbine also becomes possible. In 1994, Kubota and Nakanishi classified the unsteady flow in Pelton turbines for the study of the scale effect [1]. In 1998, Kubota et al. first proposed a numerical method called "BucFlAs" which solves the unsteady quasi-2D flow in Pelton bucket for different space/time steps individually [2]. Liu et al. described the comparative numerical analysis of the flow in buckets at different enlarging rates of jet in 2D [3]. In 2005, Zheng, Han, et al. discussed the unsteady interference between free water jet and rear surface of rotating buckets in Pelton turbine in quasi-3D[4].

So far, there is no paper reporting the unsteady water film flow in rotating Pelton bucket in 3D. In this paper, the animated cartoon approach is applied to simulate the unsteady flow in Pelton bucket. The cross section of free jet and the free curved surface is discretized by BFG. The flow particles may depart from the bucket surface because of error in simulation, it is necessary to project those particles in each moving step. A projection algorithm is proposed in this paper to solve the problem, and

*Project: Important Science and Technology Specific Projects in Zhejiang Province (Grant No. 2008C11057); Teaching Reform Research Project in Guangzhou College of SCUT (Grant No. JY110319) the example verifies the reliability of this method. Through the simulation, the visual results of free jet landing on the buckets and the unsteady flow water film on bucket surface are obtained.

\section{Discretization of Pelton Bucket and Free Jet}

\subsection{Discretization of Complicated Bucket Surface}

The boundary of Pelton bucket consists of splitter, cutout, end-wall and brim, respectively as shown in Figure 1.

Since all the boundary is curved, the orthogonal grids cannot describe the surface of Pelton bucket accurately. It is necessary to apply BFG with non-orthogonal curvilinear coordinates. The natural basis vector and their partial differential can be calculated accurately by differential geometry[5]. Those parameters are used in the algorithm of projection which will be mentioned later for CFD.

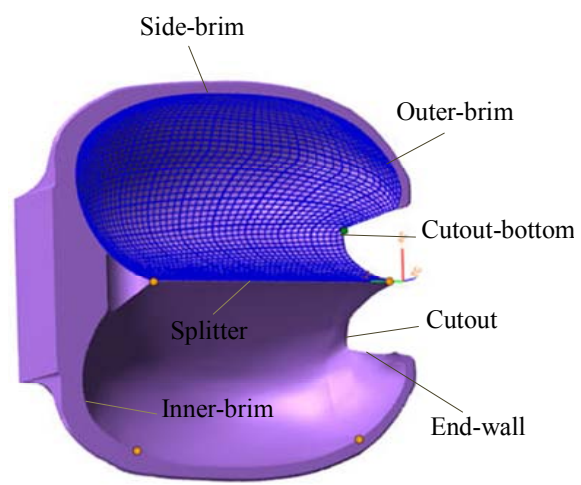

Figure 1. Complicated curved surface of Pelton bucket. 


\subsection{Discretization of the Cross Section for Free Jet}

Since the free jet is the unsteady flow with time-varying and the radius is gradually increased with the radius expansion rate. In order to simulate the unsteady flow in Pelton turbine, it is necessary to discrete the cross section of free jet into $641 \mathrm{BFG}$ considering the jet radius expansion rate as shown in Figure 2, where $R_{0}$ is the given contraction radius of jet, and $R_{r e f}$ is the reference radius of the Pelton turbine. Every jet node is defined by two parameters $j_{R}$ and $k$. Each jet cell has the attributes of four surrounding corner nodes.

\subsection{Discretization of Time and Space by iF}

The concept of analyzing the unsteady flow with the numerical method of animated cartoon approach is the application of the space-time identity number $i_{F}[6]$. It discretizes, with a short time interval, both the space and time into a series of consecutive momentary frames. In order to simulate the unsteady flow in the rotating Pelton bucket, in this paper, the spatial distance and time-step of the two adjacent buckets were evenly discretized into $N_{d i v F}=40$ under the definition of $i_{F}=0$, where the splitter tip of relevant bucket first touches to the inner-most streamline of the free jet as depicted in Figure 3.

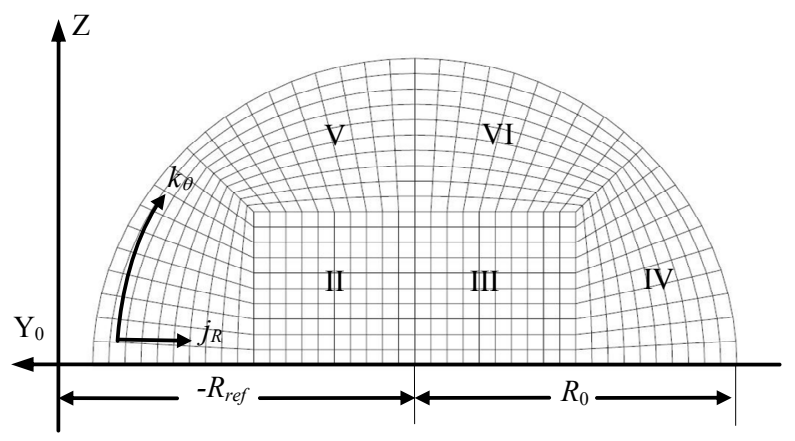

Figure 2. Discretization of cross section for free jet with butterfly BFG.

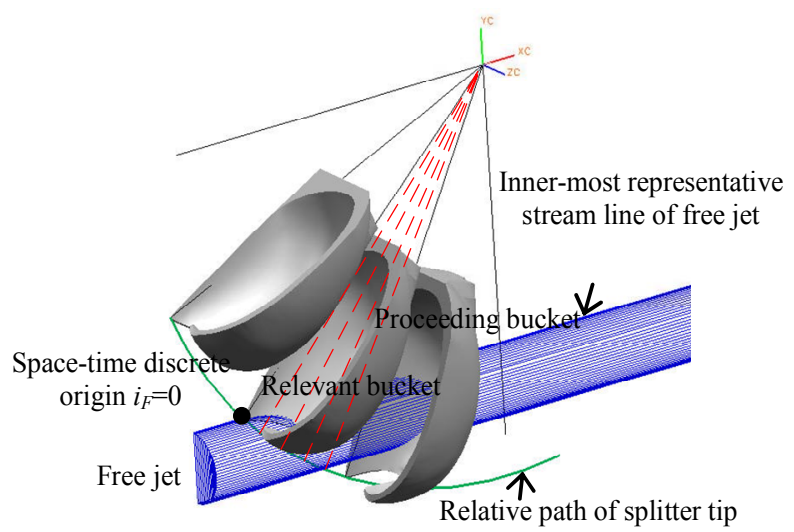

Figure 3. Discretization of time and space by frame.
So, the elapsed space-time through each discrete frame is

$$
\Delta t_{F}=2 \pi /\left(\Omega \cdot N_{B} \cdot N_{d i v F}\right)
$$

where $\Omega$ is the angular speed of the buckets, $N_{B}$ the number of buckets, and $N_{d i v F}$ the number of discrete frames in a bucket pitch.

\section{Projection of Arbitrary Position on Curved Surface}

After the free jet contacts the bucket, free jet will move freely along curved surface. Therefore, the moving grids are different from the boundary fitted grids of the bucket. The moving grids flow along the curved surface, and they may depart from the bucket surface due to the simulation error, it is necessary to project moving grids onto the bucket surface in each moving step.

So far, the curved surface of a bucket was discretized into the nodes of BFG without the attribute of surface. In order to find the arbitrary position of the curved surface, however, the curved surface of the respective local panel shall be defined as shown in Figure 4. The reliability of the BFG was clarified by the differential geometry to find the arbitrary position, in order to predict the unsteady flow on the rotating bucket for CFD.

\subsection{Projection of Spatial Point onto Curved Panel}

Let's assume that a spatial point $\mathbf{P}_{\text {pre }}\left(=X_{R p r e}{ }^{i} \mathbf{e}_{\mathrm{R}^{\prime} \mathrm{i}}\right)$ was given near to the node-C of BFG in the Cartesian frame as described in Figure 5. In order to find the position of the point $\mathbf{P}_{\text {pre }}$ on the curved surface, it is necessary to define the local curved surface of a panel by using the nearest node-C.

First, $\mathbf{P}_{\text {pre }}$ was transformed from the Cartesian frame to a natural frame at the nearest node- $\mathrm{C}$ as follows:

$$
\begin{aligned}
\Delta \mathbf{P}_{\text {pre }} & =\Delta P_{\text {pre }}^{j} \mathbf{g}_{\mathrm{Cj}} \\
& =\Delta P_{\text {pre }} \mathbf{g}_{\mathrm{C} 1}+\Delta P_{\text {pre }}{ }^{2} \mathbf{g}_{\mathrm{C} 2}+\Delta P_{\text {pre }}{ }^{3} \mathbf{g}_{\mathrm{C} 3}
\end{aligned}
$$

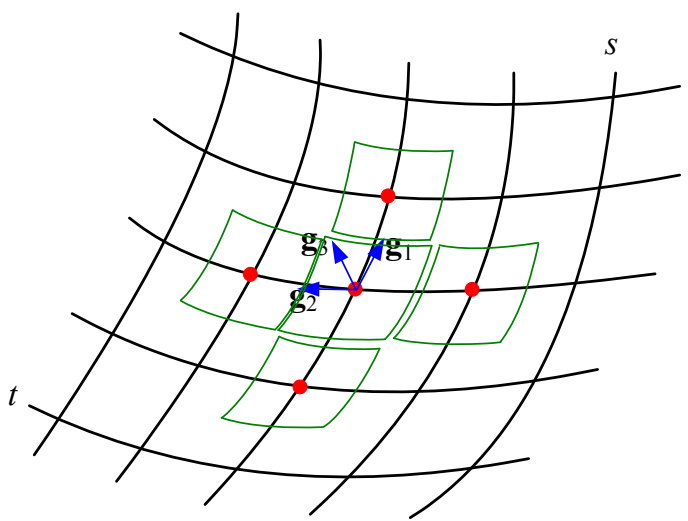

Figure 4. Local curved panel at each node. 
where $\mathbf{g}_{\mathrm{C} 1}, \mathbf{g}_{\mathrm{C} 2}$ and $\mathbf{g}_{\mathrm{C} 3}$ are the basis vectors in natural frame.

The natural component $\Delta P_{\text {pre }}^{j}$ was computed as a displacement from $\mathbf{P}_{\mathrm{C}}$ to $\mathbf{P}_{\text {pre }}$ in the local natural frame as shown in Figure 6:

$$
\begin{aligned}
\Delta P_{\text {pre }}{ }^{j} & \approx\left(\Delta P_{\text {pre }}{ }^{j} / \Delta X_{\text {pre }}{ }^{i}\right)\left(X_{\text {Rpre }}{ }^{i}-X_{R C}{ }^{\prime i}\right) \\
& =R_{C^{\prime}{ }^{j} i}\left(X_{\text {Rpre }}{ }^{i}-X_{R C}{ }^{i}\right)
\end{aligned}
$$

where $R_{C}{ }^{j}{ }_{i}$ is the matrix of transform tensor from natural frame to Cartesian frame calculating by using the cofactor $C^{\prime i}{ }_{j}$ of $R_{C}{ }^{i}{ }_{j}$ and the $\operatorname{determinant} \operatorname{det}\left(R_{C}{ }^{i}\right)$ of $R_{C}{ }^{\prime i}$ as:

$$
R_{C}{ }^{j}{ }_{i}=C^{i}{ }_{j} / \operatorname{det}\left(R_{C}{ }^{i}{ }_{j}\right)=(-1)^{{ }^{i}+\mathrm{j}} M^{\prime i} / \operatorname{det}\left(R_{C}{ }_{j}^{i}\right)
$$

where, $M_{j}^{i}$ is the minor removed the row- $j$ and the column-' $i$ from $R_{C}{ }^{i} j$ which is the matrix of transform tensor from Cartesian frame to natural frame[5].

In (2), the first two terms in the right hand side showed the projection of $\mathbf{P}_{\text {pre }}$ on the tangential flat panel $\left(\mathbf{g}_{\mathrm{C} 1} \times \mathbf{g}_{\mathrm{C} 2}\right)$, and the last term was the depth from $\mathbf{P}_{\text {pre }}$ to the projected point on the tangential flat panel as follows:

$$
\begin{aligned}
& \Delta \mathbf{P}_{\text {tan }}=\Delta P_{\text {pre }}{ }^{1} \mathbf{g}_{\mathrm{C} 1}+\Delta P_{\text {pre }}{ }^{2} \mathbf{g}_{\mathrm{C} 2} \\
& \Delta \mathbf{P}_{\text {dep }}=\Delta P_{\text {pre }}{ }^{3} \mathbf{g}_{\mathrm{C} 3}
\end{aligned}
$$

The height $\Delta P_{\text {high }}{ }^{3}$ from the $\mathbf{P}_{\text {tan }}$ on the tangential flat panel to the curved panel of bucket was predicted by the differential geometry as shown in Figure 7 as:

$$
\begin{gathered}
\Delta \mathbf{P}_{\text {high }}=\Delta P_{\text {high }}{ }^{3} \mathbf{g}_{\mathrm{C} 3} \\
\Delta P_{\text {high }}{ }^{3}= \\
(1 / 2)\left\{L_{C}\left(\Delta P_{\text {pre }}\right)^{1}\right. \\
\left.+\left(M_{C 1}+M_{C 2}\right) \Delta P_{\text {pre }}{ }^{1} \Delta P_{\text {pre }}{ }^{2}+N_{C}\left(\Delta P_{\text {pre }}{ }^{2}\right)^{2}\right\}
\end{gathered}
$$

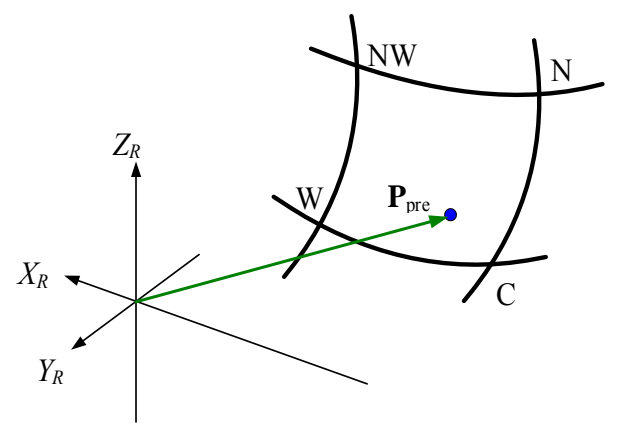

Figure 5. $P_{\text {pre }}$ surrounded by four nodes including node-C.

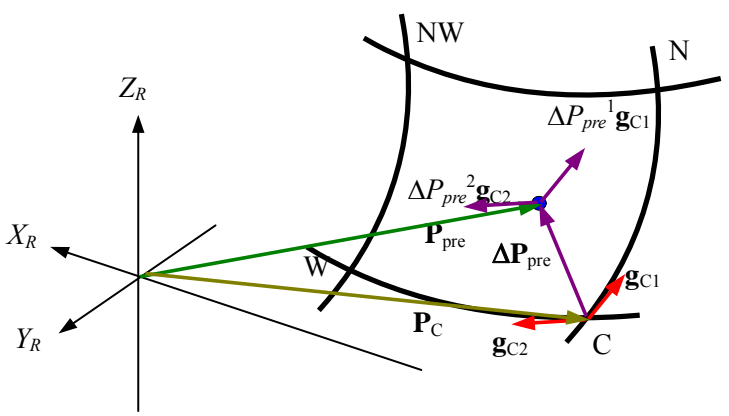

Figure 6. $\Delta \mathrm{P}_{\text {pre }}$ defined by natural frame at node-C. where, $L_{C}, M_{C 1}, M_{C 2}$ and $N_{C}$ can be computed by differential geometry[5].

In conclusion, the position $\Delta \mathbf{P}_{\text {post }}$ on the curved panel was determined as a point projected from $\Delta \mathbf{P}_{\text {pre }}$ onto the tangential flat panel based on the node- $\mathrm{C}$ in the natural frame as follows:

$$
\begin{aligned}
\Delta \mathbf{P}_{\text {post }} & =\Delta \mathbf{P}_{\text {tan }}+\Delta \mathbf{P}_{\text {high }} \\
& =\Delta P_{\text {pre }}{ }^{1} \mathbf{g}_{\mathrm{C} 1}+\Delta P_{\text {pre }}{ }^{2} \mathbf{g}_{\mathrm{C} 2}+\Delta P_{\text {high }}{ }^{3} \mathbf{g}_{\mathrm{C} 3}
\end{aligned}
$$

\subsection{Verification of Prediction}

In order to prove the reliability of this algorithm of projection, we make a comparison for the moving grids between projection and without projection. Figure 8 shows the result of comparison at $i_{F}=5$. Figure 8(a) clearly shows that some moving grids are departing from the boundary surface of the bucket without using the projection. The water film flows along the boundary surface of buckets, it is just close to the surface but not departing from the surface. All the moving grids are close to the curved surface of buckets after using the algorithm of projection as shown in Figure 8(b). Therefore, this projection algorithm is necessary and reliable for the unsteady simulation of flow particles in Pelton bucket.

\section{Simulation Result}

A Pelton turbine having the geometrical specific speed $B / D_{\text {ref }}=0.35$ with 18 buckets was selected as the numerical model for the unsteady flow investigation. Under the optimum unit speed $n_{D H}=40 \mathrm{rpm}$, the ratio of splitter

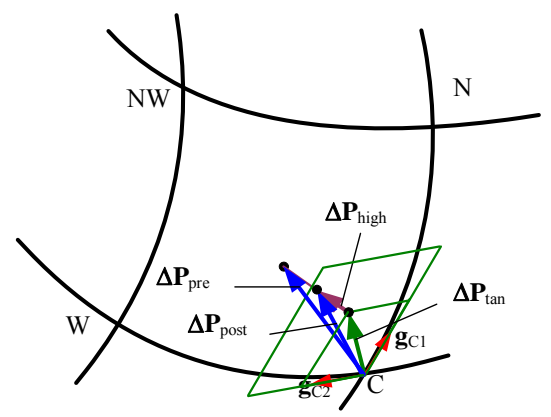

Figure 7. Height from tangential flat panel to curved surface.

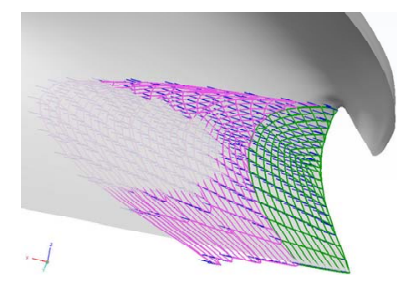

(a) Before projection at $i_{F}=5$

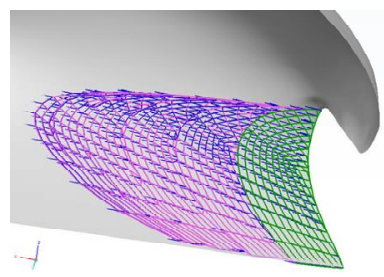

(b) After projection at $i_{F}=5$
Figure 8. Comparison of water sheet before and after projection at $i_{F}=5$. 
tip speed $=1.4$. The expansion rate of jet radius was assumed as $k_{R j}=0.1 \%$. Consider the cartoon frames between two adjacent buckets $N_{d i v F}=40$, discretization number along $j_{R}$ direction $N_{d i v R 0}=40$, and discretization number along $k$ direction $N_{d i v \theta 0}=20$.

\subsection{Visualization of Landing Jet on Inner Surface of Bucket}

The block diagram of jet landing on bucket surface realized by CFD was shown in Figure 9.

By using the algorithm in the above block diagram, the jet nodes landing on bucket surface were simulated numerically in 3D as shown in Figure 10 for different frames.

At $i_{F}=0$ where is the origin of the discretization in space and time, outer margin of the free jet just touch the splitter tip of the bucket. Since the shape of the cutout is complicated, there is a little water entering the bucket. Free jet entering the bucket is increasing with $i_{F}$ increased, until the whole jet enters the bucket. At $i_{F}=40$, the whole bucket has already entered the rotating bucket. After $i_{F}=40$, the following bucket starts touching and intercepting the free jet. From $i_{F}=90$, it is no longer the whole jet entering the relevant bucket. The jet entering the bucket is less and less with the continuous increasing of $i_{F}$. From $i_{F}=128$, there is no more jet entering the bucket. The visualization of numerical results meets the flow phenomena in Pelton bucket; and they are very useful to study the mysterious flow in Pelton bucket.

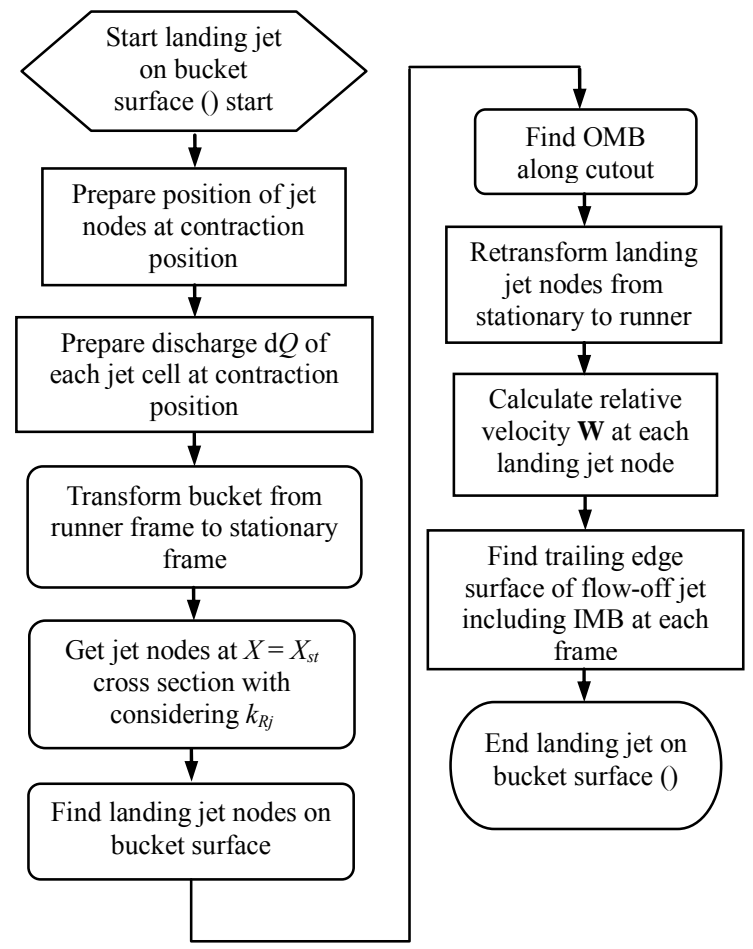

Figure 9. Block diagram for finding landing jet on surface.
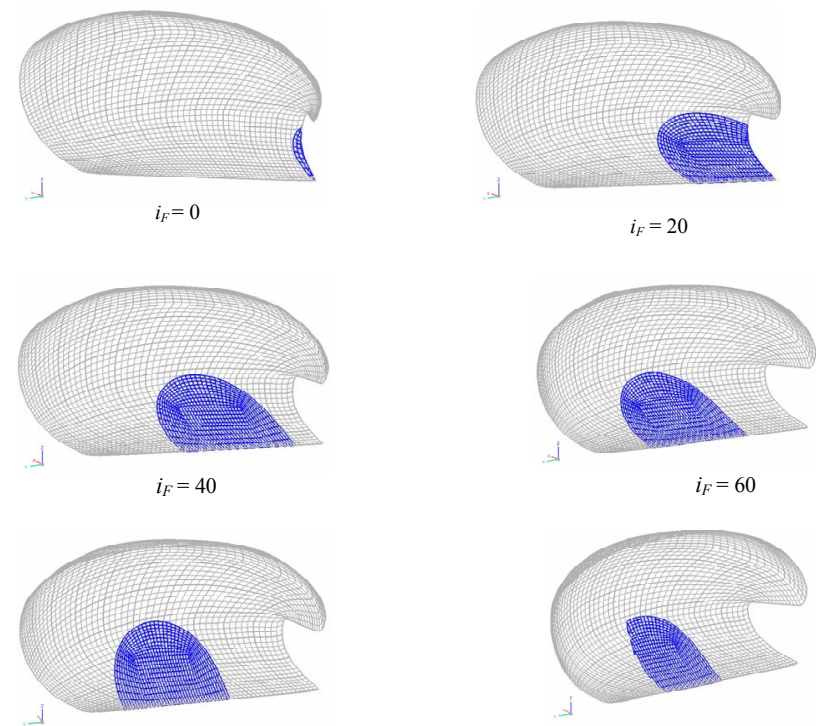

$i_{F}=80$

$i_{F}=100$

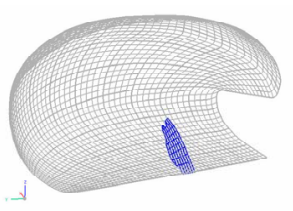

$i_{F}=120$

$i_{F}=127$

Figure 10. Landing jet on surface at different frames.

\subsection{Visualization of Water Film on Rotating Bucket}

According to the numerical setting up above, the simulation result of the water film flowing on the rotating bucket is obtained. As shown in Figure 11, the free jet is increasing with $i_{F}$ increased. The water film in the rotating bucket flows towards to the out-brim of the bucket orderly. This simulation result can supply the powerful technical support for the optimization design of the Pelton bucket.

\section{Conclusions}

The complicated unsteady flow is simulated by the animated cartoon approach, and we can draw out following conclusions:

1) The curved surface of a bucket is described by BFG with non-orthogonal curvilinear coordinates and the cross section of the free jet is discrete with butterfly BFG for CFD.

2) The moving grids are successfully projected onto the curved bucket's surface by a projection algorithm proposed in the paper.

3) The visualization result of the jet landing on bucket's surface and the unsteady flow in the rotating buckets in $3 \mathrm{D}$ verifies the effectiveness of the proposed 


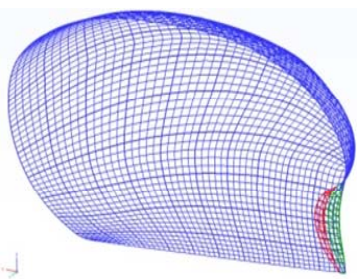

$i_{F}=1$

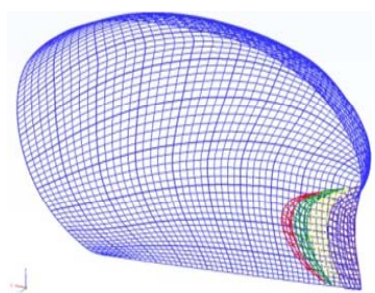

$i_{F}=3$

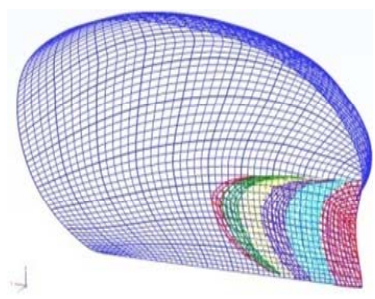

$I_{F}=5$

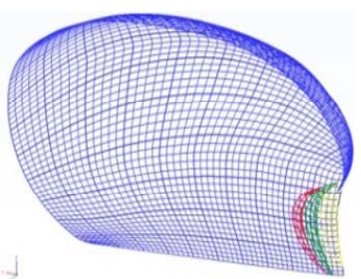

$i_{F}=2$

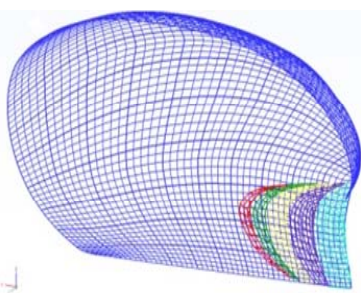

$i_{F}=4$

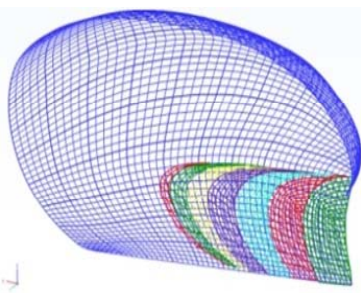

$i_{F}=6$
Figure 11. Unsteady water film at different frames.

method. The simulation result can supply the powerful technical support for the optimization design of the Pelton bucket.

\section{REFERENCES}

[1] T. Kubota and Y. Nakanishi, "Classification of Flow in Pelton Turbines for Study of Scale Effect,"17th IAHR-Symposium, Beijing, Vol. G-6, 1994, pp. 865-876.

[2] T. Kubota, J. Xia, H. Takeuchi, et al., "Numerical Analysis of Free Water Sheet Flow on Pelton Buckets," Proceedings of 19th IAHR Symposium, Singapore, 1998, pp. 316-329.

[3] J. Liu, C. X. Wei and F. Q. Han, "Effect of Enlarged Free Jet on Energy Conversion in Pelton Turbine," Journal of Hydrodynamics, Series B, Vol. 18, No. 2, 2006, pp. 211-218.

[4] A. L. Zheng, F. Q. Han, Y. X. Xiao, et al., "Unsteady Interference between Jet and Rear Surface of Rotating Buckets in Pelton Turbine," 8th Asian International Fluid Machinery Conference, Yichang, China, 2005, pp. 302-310.

[5] N. Shen and T. Kubota, "Curved Surface of Pelton Bucket Based on Differential Geometry,"APPEEC2012, Shanghai, Vol. 3, 2012.

[6] F. Q. Han, N. Shen, L. X. Li, et al., "Unsteady Separation of Jet Branch by Cutout of Rotating Pelton Bucket," SCIENCE CHINE Technological Science, Vol. 54, No. 2, 2011, pp. 302-310. doi: 10.1007/s11431-010-4263-2 